\title{
Precoding for Coded Communication on Block Fading Channels and Cooperative Communications
}

\author{
Dieter Duyck, Joseph J. Boutros, and Marc Moeneclaey
}

\begin{abstract}
We study precoding for the outage probability minimization of block fading (BF) channels and BF relay channels. Recently, an upper bound on the outage probability with precoding was established for BF channels, but only for high instantaneous SNR. This upper bound is much easier to minimize than the actual outage probability, so that optimal precoding matrices can be determined without much computational effort. Here, we provide a proof for the upper bound on the outage probability at low instantaneous SNR. Next, the structure of the precoding matrix is simplified so that it can be easily constructed for an arbitrary number of blocks in the BF channel. Finally, we apply this technique to cooperative communications.
\end{abstract}

\section{INTRODUCTION}

A block fading (BF) channel [2] with $B$ blocks is a useful model for all channels that consist of parallel subchannels (e.g. via time-interleaving, frequency hopping, HARQ, OFDMA or cooperative communications). The outage probability limit [2], [13] is a fundamental and achievable lower bound on the average word error rate (WER) of coded systems without channel state information at the transmitter (no CSIT). By choosing a well designed precoding matrix $P$, the outage probability can be minimized. This minimization requires a multivariate 1 brute force optimization, involving a Monte Carlo simulation 2 for each evaluation of the outage probability, which is intractable. The minimum product distance approach, proposed in [1], [3] and extended by e.g. [12] for MIMO, is not suitable for coded schemes because it optimizes the bit error rate of uncoded constellations transmitted on ergodic fading channels. Fortunately, a useful upper bound on the outage probability was established [6], [5], which can be optimized with a small computational effort (a few seconds with the current computer resources). The proof for this upper bound was given in [6] for high instantaneous SNR only.

Here, we analyse this upper bound and show that the proof for high instantaneous SNR is not sufficient. Next, a construction method for the precoding matrix $P$ is lacking when $B \geq 3$. We provide a proof for low SNR and we elaborate on

Dieter Duyck and Marc Moeneclaey are with the Department of Telecommunications and Information processing, Ghent University, StPietersnieuwstraat 41, B-9000 Gent, Belgium, \{dduyck,mm\} @ telin.ugent.be.

Joseph J. Boutros is with Texas A\&M University at Qatar, PO Box 23874 Doha, Qatar, boutros@tamu.edu

(C) 2011 IEEE. Personal use of this material is permitted. Permission from IEEE must be obtained for all other uses, in any current or future media, including reprinting/republishing this material for advertising or promotional purposes, creating new collective works, for resale or redistribution to servers or lists, or reuse of any copyrighted component of this work in other works.

${ }^{1} \mathrm{~A}$ unitary $B \times B$ precoding matrix has $B^{2}$ degrees of freedom.

${ }^{2}$ Monte Carlo simulations are required to take into account the distribution of all fading gains. the structure of $P$ so that it can be easily constructed for all $B$. The results can be applied to cooperative communications, which is shown in the last section. The work presented here is partly covered in [7], but new material such as the necessity of a new proof for low SNR and the application to cooperative communications is added.

\section{A NEW CHANNEL EQUATION FOR BF CHANNELS}

The transmitter output is a real or complex vector $\mathbf{x}=$ $[\mathbf{x}(1), \ldots \mathbf{x}(B)]$ where $\mathbf{x}(b)=\left[x(b)_{1}, \ldots, x(b)_{\frac{N}{B}}\right]$ is the $b$ th part of the transmitted vector. The received vector and the noise vector are similarly represented. The channel is memoryless with additive white Gaussian noise and multiplicative real fading (Rayleigh distributed). The fading coefficients are only known at the decoder side (no CSIT) where the received signal vector is $\mathbf{y}(b)=\alpha_{b} \mathbf{x}(b)+\mathbf{w}(b), \quad b=1, \ldots, B$, where the fading coefficient $\alpha_{b}$ is independent and identically distributed (i.i.d.) from block to block. The noise vector $\mathbf{w}(b)$ consists of $N / B$ independent noise samples which are complex Gaussian distributed, $w(b)_{n} \sim \mathcal{C N}\left(0, \frac{1}{\gamma}\right)$, where $\gamma$ is the average SNR.

The transmitter output is obtained after component interleaving combined with linear precoding [3], [8]. In our case, each string of $m$ bits is mapped to one of $M=2^{m}$ points belonging to a B-dimensional real or complex space; the corresponding B-dimensional M-point constellation $\Omega_{z}$ is denoted $M-\mathcal{R}^{B}$ or $M-\mathcal{C}^{B}$, respectively. Denoting as $\mathbf{z}_{n}=$ $\left[z(1)_{n}, \ldots, z(B)_{n}\right]^{T}$ the B-dimensional vector that results from mapping the $\mathrm{n}$-th string of $m$ coded bits, the linear precoding involves the computation

$$
\mathbf{x}_{n}=P \mathbf{z}_{n}, n=1, \ldots, \frac{N}{B},
$$

where the symbols $\left\{x(b)_{n}, b=1, \ldots, B\right\}$ are the components of the multidimensional symbol $\mathbf{x}_{n}$ (we similarly consider $\mathbf{y}_{n}$ and $\mathbf{w}_{n}$ ), which belongs to the $B$-dimensional $M$-point constellation $\Omega_{x}$.

A precoding matrix $P$ that is unitary is an obvious choice because it does not decrease the capacity of a Gaussian channel. In this paper, we restrict our study to real precoding matrices, hence $P$ is orthogonal. When $B=2, P$ is a rotation matrix where the rotation angle $\theta$ is the degree of freedom. However, rotation matrices are difficult to construct for higher dimensions. In Sec. III it is shown that for $B>2$ it is sufficient to consider orthogonal circulant precoding matrices. We denote its first row as $\left(p_{0}, \ldots, p_{B-1}\right)$. The second row is a cyclic shift to the right of the first row, and so on. Because the columns of the $B \times B$ Fourier matrix $F$ are the eigenvectors 


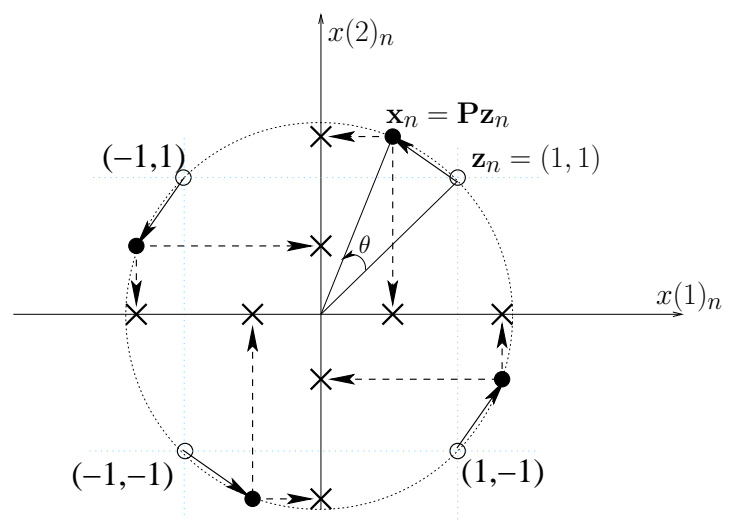

Fig. 1. Displaying the rotation at the transmitter for $B=2$. The empty (filled) circles represent $\Omega_{z}\left(\Omega_{x}\right)$. The components of $\mathbf{t}_{n}$ can be obtained by scaling the components $\mathbf{x}_{n}$ by their respective fading gain. The crosses on the coordinate axes are the transmitted vector components.

of any circulant matrix, we can construct $P$ as follows:

$$
P=F \Lambda F^{H},
$$

where $(F)_{m, n}=\frac{1}{\sqrt{B}} \exp \left(\frac{-2 j \pi m n}{B}\right), m, n \in\{0, \ldots, B-1\}$, and $\Lambda$ is a diagonal matrix containing the eigenvalues of $P$. The condition for $P$ being orthogonal is $\Lambda^{H} \Lambda=I_{B}$, or the $B$ eigenvalues of $P$ must have a squared magnitude of 1 . It is easy to find that

$$
\lambda_{n}=\sum_{l=0}^{B-1} p_{l} \exp \left(\frac{-j 2 \pi n l}{B}\right) .
$$

Now, it follows that

$$
p_{l}=\frac{1}{B} \sum_{m=0}^{B-1} \lambda_{m} \exp \left(\frac{j 2 \pi m l}{B}\right) .
$$

As the eigenvalues must have a magnitude of 1 , we have $\lambda_{n}=\exp \left(j \theta_{n}\right)$. In order to obtain a real-valued $P$, we take $\lambda_{0} \in\{ \pm 1\}$ and $\lambda_{B-n}=\left(\lambda_{n}\right)^{*}$ (i.e., $\theta_{B-n}=-\theta_{n}$ ) for $n=$ $1, \ldots, B-1$. For $B>2, P$ is determined by $\lfloor(B-1) / 2)\rfloor$ continuous parameters that can be optimized.

Fig. 1 illustrates the effect of precoding for $B=2$ when a 4- $\mathcal{R}^{2}$ constellation is used as $\Omega_{z}$. In that case, $\Omega_{x}$ is a rotated version of $\Omega_{z}=4-\mathcal{R}^{2}$. When received at the destination, the components of $\mathbf{x}_{n}$ are affected by their corresponding fading gain and noise. Consider the vector $\mathbf{t}_{n}=\boldsymbol{\alpha} \cdot \mathbf{x}_{n}=$ $\left[\alpha_{1} x(1)_{n}, \ldots, \alpha_{B} x(B)_{n}\right]$, which belongs to the constellation $\Omega_{t}$, which we denote as the faded constellation. Note that this constellation is different for each codeword, because the fading point $\boldsymbol{\alpha}$ changes.

This system is better modelled by the channel equation

$$
\mathbf{y}_{n}=\mathbf{t}_{n}+\mathbf{w}_{n}, \quad n=1, \ldots, \frac{N}{B} .
$$

Depending on the set of fading points $\alpha$ that is considered, the constellation $\Omega_{t}$ at the input of this Gaussian vector channel varies. This new channel equation gives more insight and is important in the new proofs proposed here.

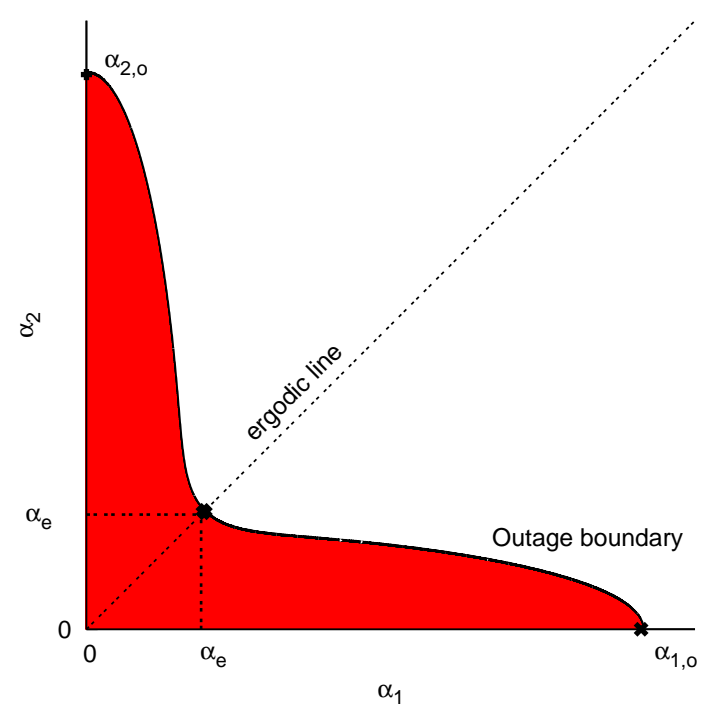

Fig. 2. The outage boundary limits the region $V_{o}$ (coloured red) in the fading space which corresponds to an information theoretic outage event. The points $\left\{\boldsymbol{\alpha}_{b, o}, b=1, \ldots, B\right\}$ and $\boldsymbol{\alpha}_{e}$ are shown for $B=2, \Omega_{z}=4-\mathcal{R}^{2}$ and $\theta=10^{\circ}$. The information rate is $R=0.9$ bpcu. The average SNR is fixed to $\gamma=8 \mathrm{~dB}$.

\section{Method to Upper Bound Outage Probability}

For the remainder of the paper, we drop the index $n$ in the vectors $\mathbf{z}_{n}, \mathbf{x}_{n}, \mathbf{t}_{n}, \mathbf{y}_{n}$ and $\mathbf{w}_{n}$, as the time index is not important when considering mutual information. We write random variables using upper case letters corresponding to the lower case letters used for their realizations. The mutual information $I(\boldsymbol{\alpha}, \gamma, P)$ at a certain fading point $\boldsymbol{\alpha}$ between the transmitted $B$-dimensional symbol $\mathrm{x}$ and the corresponding received vector $\mathbf{y}$ is given by [8],

$$
I\left(\boldsymbol{\alpha}, \gamma, P, \Omega_{z}\right)=\frac{1}{B} I(\mathbf{X} ; \mathbf{Y} \mid \boldsymbol{\alpha}, \gamma)=\frac{1}{B} I(\mathbf{T} ; \mathbf{Y} \mid \boldsymbol{\alpha}, \gamma),
$$

where the last term corresponds with Eq. (5), and where the scaling factor $\frac{1}{B}$ is added because the $B$ blocks in the channel timeshare a time-interval.

The outage probability is the probability that the instantaneous mutual information is less than the transmitted rate. In other words, the outage probability corresponds to the set of fading gains where the channel is sufficiently bad (the channel is in outage):

$$
P_{\text {out }}\left(\gamma, \Omega_{z}, P, R\right)=\int_{\boldsymbol{\alpha} \in V_{o}} p(\boldsymbol{\alpha}) d \boldsymbol{\alpha},
$$

where $V_{o}$ is the region of fading gains in the fading space [4] such that $I\left(\boldsymbol{\alpha}, \gamma, P, \Omega_{z}\right)<R$. The region $V_{o}$ is limited by an outage boundary $B_{o}\left(\gamma, P, \Omega_{z}, R\right)$, defined by $I\left(\boldsymbol{\alpha}, \gamma, P, \Omega_{z}\right)=R$ (Fig. 2).

Definition 1: We define $\alpha_{b, o}$ by the magnitude of the intersection between the outage boundary and the axis $\alpha_{b}$. More precisely, $I\left(\left.\boldsymbol{\alpha}\right|_{\alpha_{i}=0, i \neq b, \alpha_{b}=\alpha_{b, o}}, \gamma, P, \Omega_{z}\right)=R$.

Definition 2: We define $\alpha_{e}$ as the value of the components of the intersection between the outage boundary and the line $\alpha_{1}=\ldots=\alpha_{B}$ (also known as the ergodic line). More precisely, $I\left(\left.\boldsymbol{\alpha}\right|_{\alpha_{i}=\alpha_{e}, i \in[1, \ldots, B]}, \gamma, P, \Omega_{z}\right)=R$.

In the remainder of the paper, we denote the points 
$\left.\boldsymbol{\alpha}\right|_{\alpha_{i}=0, i \neq b, \alpha_{b}=\alpha_{b, o}}$ by $\boldsymbol{\alpha}_{b, o}$ and $\left.\boldsymbol{\alpha}\right|_{\alpha_{i}=\alpha_{e}, i \in[1, \ldots, B]}$ by $\boldsymbol{\alpha}_{e}$. The defined points are illustrated in Fig. 2 for $B=2$.

In the following proposition, we determine a sufficient condition for the points $\boldsymbol{\alpha}_{b, o}$ to have the same magnitude.

Proposition 1: On a BF channel with $B=2$, with a discrete input alphabet and with linear precoding, the magnitudes $\left\{\alpha_{b, o}, b=1,2\right\}$ are equal if the constellation is invariant under a rotation of $90^{\circ}$ and the precoding matrix is orthogonal.

On a BF channel with $B>2$, with a discrete input alphabet and with linear precoding, the magnitudes $\left\{\alpha_{b, o}, b=\right.$ $1, \ldots, B\}$ are equal if the constellation is invariant under a cyclic shift of the components of the points of the constellation and the precoding matrix is an orthogonal circulant matrix.

Proof: The points $\left\{\boldsymbol{\alpha}_{b, o}, b=1, \ldots, B\right\}$ correspond to the case that all fading gains are zero, except one, whose value is the scaling factor of the projection of the multidimensional constellation on the $\mathrm{b}$-th coordinate axis $x(b)$, so that the mutual information between the $\mathbf{X}$ and $\mathbf{Y}$ is equal to the spectral efficiency $B R$. In other words, if the projection of the multidimensional constellation on each coordinate axis yields the same set of points, then the magnitudes of the points $\left\{\boldsymbol{\alpha}_{b, o}, b=1, \ldots, B\right\}$ are equal.

First, we restrict our attention to the case that $B=2$. Consider the constellation point $\mathbf{z}^{(i)}=\left(u^{(i)}(1), u^{(i)}(2)\right) \in$ $\Omega_{z}$. The projection of the multidimensional constellation on each coordinate axis yields the same set of points if for each point $\mathbf{z}^{(i)}$, the points $\mathbf{z}^{(j)}=\left(u^{(j)}(1), u^{(j)}(2)\right)$ and $\mathbf{z}^{(q)}=\left(u^{(q)}(1), u^{(q)}(2)\right)$ exist, $i, j, q \in\left[1, \ldots, 2^{m}\right] ; j, q \neq i$, so that

$\left\{\begin{array}{c}\cos (\theta) u^{(i)}(1)-\sin (\theta) u^{(i)}(2)=\sin (\theta) u^{(j)}(1)+\cos (\theta) u^{(j)}(2) \\ \sin (\theta) u^{(i)}(1)+\cos (\theta) u^{(i)}(2)=\cos (\theta) u^{(q)}(1)-\sin (\theta) u^{(q)}(2) .\end{array}\right.$

In other words, $x^{(i)}(1)=x^{(j)}(2)$ and $x^{(i)}(2)=x^{(q)}(1)$, where $\mathbf{x}^{(i)}, \mathbf{x}^{(j)}$ and $\mathbf{x}^{(q)}$ are the corresponding points of $\mathbf{z}^{(i)}, \mathbf{z}^{(j)}$ and $\mathbf{z}^{(q)}$ in $\Omega_{x}$. It can be easily verified that this is always fulfilled if

$$
\left\{\begin{array}{l}
\left(u^{(i)}(1), u^{(i)}(2)\right)=\left(u^{(j)}(2),-u^{(j)}(1)\right) \\
\left(u^{(i)}(1), u^{(i)}(2)\right)=\left(-u^{(q)}(2), u^{(q)}(1)\right),
\end{array}\right.
$$

or in other words, the constellation is invariant under a rotation of $\pi / 2$, which proves what was claimed.

Now consider the case that $B>2$. Consider the $B$ dimensional constellation $\Omega_{z}$ that contains $M$ points. When $\mathbf{z}$ belongs to $\Omega_{z}$, then also $\mathbf{z}^{(1)}, \ldots, \mathbf{z}^{(B-1)}$ belong to $\Omega_{z}$, where $\mathbf{z}^{(b)}$ is obtained from $\mathbf{z}$ by a $b$-fold upward cyclic shift of the components of $\mathbf{z}: \mathbf{z}^{(b)}=C^{b} \mathbf{z}$, where $C$ is obtained as a cyclic shift to the right of the columns of the $B \times B$ identity matrix. Note that the number of constellation points does not need to be a multiple of $B$ : a subset of the constellation may consist of an arbitrary number of constellation points of the type $[z, z, \ldots, z]^{T}$ which remain invariant under a cyclic shift.

Consider an orthogonal circulant $B \times B$ precoding matrix $P$. Therefore, $P=C P C^{T}$ (a circulant matrix remains the same when applying a left cyclic shift to the columns and an upward cyclic shift to the rows). The transformation of $\mathbf{z}^{(b)}$ is

$$
P \mathbf{z}^{(b)}=P C^{b} \mathbf{z}=\left(C P C^{T}\right) C^{b} \mathbf{z}=\ldots=C^{b} P \mathbf{z}=\mathbf{x}^{(b)},
$$

where we exploit that $C$ is an orthogonal matrix.

Consider the matrix $\left(\mathbf{x}, \mathbf{x}^{(1)}, \ldots, \mathbf{x}^{(B-1)}\right)$. As the $(i+1)$-th row is obtained as a cyclic shift to the left of the $i$-th row, the set of components in a row is the same for each row. A constellation point in $\Omega_{z}$ of the type $(z, z, \ldots, z)^{T}$ is transformed into a constellation point in $\Omega_{x}$ of the type $(x, x, \ldots, x)^{T}$. We conclude that the projection of the constellation $\Omega_{x}$ on any of the coordinate axes yields the same set of points.

In the remainder of this paper, it is assumed that the constellation used at the transmitter fulfils Prop. 1. The magnitudes $\left\{\alpha_{b, o}, b=1, \ldots, B\right\}$ are then simply denoted by $\alpha_{o}$, and the points $\boldsymbol{\alpha}_{b, o}$ by $\boldsymbol{\alpha}_{o}$. This also means that the projection of $\Omega_{x}$ on either coordinate axes yields the same set of points, which we denote by $\mathcal{S}_{p}$, where $p$ stands for projection. Multidimensional constellations fulfilling Prop. 1 have an interesting property: $I\left(X(b) ; Y(b) \mid \alpha_{b}=\alpha, \gamma\right)$ does not depend on $b$. As a consequence, we represent this mutual information by $I_{\mathcal{S}_{p}}\left(\alpha^{2} \gamma, P, \Omega_{z}\right)$.

From Eq. (7), it is clear that the outage probability can be upper bounded by $P_{u p}$ corresponding to a boundary $B_{u p}$, outer bounding $B_{o}\left(\gamma, P, \Omega_{z}, R\right)$ :

$$
P_{u p}=\int_{\boldsymbol{\alpha} \in V_{u p}} p(\boldsymbol{\alpha}) d \boldsymbol{\alpha},
$$

where $V_{u p}$ is the region limited by $B_{u p}$. In [6], a boundary with a simple shape outer bounding $B_{o}\left(\gamma, P, \Omega_{z}, R\right)$ was determined, which is then much easier to optimize. A surface in the fading space, $U(\boldsymbol{\alpha})=0$, outer bounds $B_{o}\left(\gamma, P, \Omega_{z}, R\right)$ if and only if

$$
I\left(\boldsymbol{\alpha}, \gamma, P, \Omega_{z}\right) \geq R, \text { for all } \boldsymbol{\alpha} \text { satisfying } U(\boldsymbol{\alpha})=0 .
$$

Here, the new channel equation, Eq. (5), is used. By showing that for all $\boldsymbol{\alpha}$ satisfying $U(\boldsymbol{\alpha})=0$, the constellation $\Omega_{t}$ is distorted in such a way that $I(\mathbf{T} ; \mathbf{Y} \mid \boldsymbol{\alpha}, \gamma) \geq B R$, it is proved that $U(\boldsymbol{\alpha})=0$ outer bounds $B_{o}\left(\gamma, P, \Omega_{z}, R\right)$.

For example, for general $B$ and for high instantaneous SNR, it is proved in [6] that a $B$-hypersphere touching the outage boundary on the axes of the fading space, hence with radius $\alpha_{o}$, outer bounds $B_{o}\left(\gamma, P, \Omega_{z}, R\right)$. A $B$-hypersphere $U(\boldsymbol{\alpha})=$ 0 is a generalization of a sphere to $B$ dimensions,

$$
U(\boldsymbol{\alpha})=\sum_{b=1}^{B} \alpha_{b}^{2}-\alpha_{o}^{2}
$$

The proof was based on the approximation of the mutual information $I(\mathbf{T} ; \mathbf{Y} \mid \boldsymbol{\alpha}, \gamma)$ at high SNR [11], [14

$$
I(\mathbf{T} ; \mathbf{Y} \mid \boldsymbol{\alpha}, \gamma) \approx m-2 \frac{K \pi}{2^{m} d_{m i n}^{2}(\boldsymbol{\alpha})} Q\left(d_{m i n}(\boldsymbol{\alpha}) \sqrt{\gamma / 2}\right)
$$

where $d_{\min }(\boldsymbol{\alpha})$ is the minimal distance of the constellation $\Omega_{t}$ and $K$ is the number of pairs of points at minimum distance in the constellation $\Omega_{t}$. The SNR considered in [11], [14] is $\frac{\mathbb{E}\left[\left|\mathbf{t}^{2}\right|\right]}{\mathbb{E}\left[\left|\mathbf{w}^{2}\right|\right]}=\gamma|\boldsymbol{\alpha}|^{2}$. Because only the fading gains on the hypersphere (11) are considered, we have $|\boldsymbol{\alpha}|^{2}=\alpha_{o}^{2}$. From the definition of $\alpha_{o}$ (Def. 1), $\gamma \alpha_{o}^{2}$ is constant, so that $I\left(\mathbf{T} ; \mathbf{Y} \mid \boldsymbol{\alpha}=\boldsymbol{\alpha}_{o}, \gamma\right)=I_{\mathcal{S}_{p}}\left(\alpha_{o}^{2} \gamma, P, \Omega_{z}\right)=B R$. For example, in Fig. 3, we show the mutual information $\frac{I_{\mathcal{S}_{p}}\left(\alpha^{2} \gamma, P, \Omega_{z}\right)}{B}$ corresponding with $\Omega_{z}=8-\mathcal{R}^{2}$. It is clear that $\gamma \alpha_{o}^{2}$ is between 


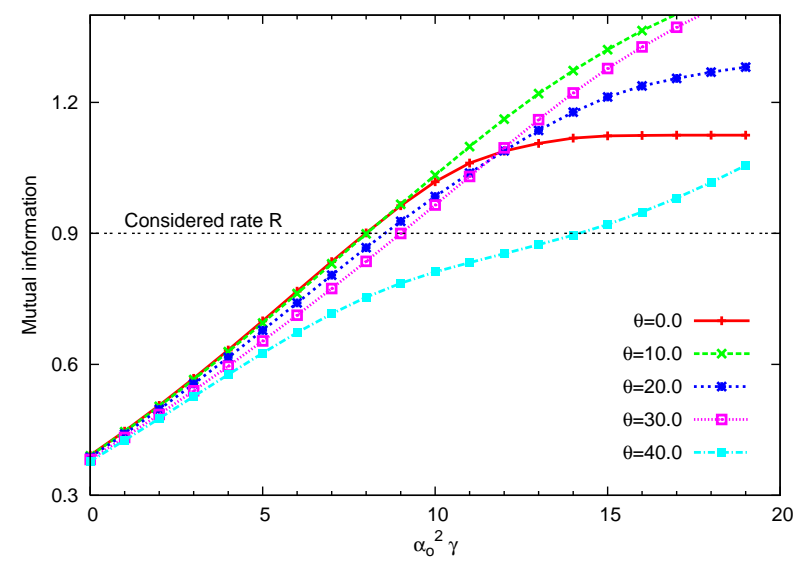

Fig. 3. The mutual information $I\left(\boldsymbol{\alpha}=\boldsymbol{\alpha}_{o}, \gamma, P\right)=\frac{I_{\mathcal{S}_{p}}\left(\alpha_{o}^{2} \gamma, P\right)}{B}$ corresponding with $\Omega_{z}=8-\mathcal{R}^{2}$ is shown for each instantaneous SNR $\gamma \alpha_{o}^{2}$. The rate $R=0.9$ is achieved for $\alpha_{o}^{2} \gamma \in[8,14] \mathrm{dB}$, depending on the rotation angle $\theta$.

$8 \mathrm{~dB}$ and $14 \mathrm{~dB}$, depending on the rotation angle $\theta$. Two SNR regimes are visible in the mutual information, one for high SNR and one for low SNR, in which the optimal rotation angles are different. The appropriate regime depends on the considered rate $R$. So limiting the analysis of $I(\mathbf{T} ; \mathbf{Y} \mid \boldsymbol{\alpha}, \gamma)$ to the high SNR regime, as in Eq. (12), is not sufficient in general. Therefore, we provide an additional proof for low SNR in the next section.

\section{An Upper Bound on the Outage Probability}

Here, we show that also for low instantaneous $\operatorname{SNR}\left(\gamma|\boldsymbol{\alpha}|^{2}\right)$, the outage boundary is outer bounded by the hypersurface of a $B$-hypersphere touching it at $\boldsymbol{\alpha}_{o}$. Note that $\gamma|\boldsymbol{\alpha}|^{2} \rightarrow 0$ if and only if $\gamma \alpha_{b}^{2} \rightarrow 0, \forall b=1, \ldots, B$.

Proposition 2: On a BF channel at low instantaneous SNR, with a discrete input alphabet and with linear precoding, the outage boundary $B_{o}\left(\gamma, P, \Omega_{z}, R\right)$ is outer bounded by the hypersurface of the $B$-hypersphere $\alpha_{1}^{2}+\alpha_{2}^{2}+\ldots+\alpha_{B}^{2}=\alpha_{o}^{2}$ touching it at the axes of the fading space.

Proof: Consider the mutual information $I(\boldsymbol{\alpha}, \gamma, P)$ of the constellation $\Omega_{t}$, given the fading gains $\alpha$ (Eq. [6) [8]:

$$
I\left(\boldsymbol{\alpha}, \gamma, P, \Omega_{z}\right)=\frac{m}{B}-\frac{2^{-m}}{B} \sum_{\mathbf{x} \in \Omega_{x}} \mathbb{E}_{\mathbf{y} \mid \mathbf{x}}\left[\log _{2}\left(f\left(\boldsymbol{\alpha}, \Omega_{x}, \mathbf{y}\right)\right)\right]
$$

where $f\left(\boldsymbol{\alpha}, \Omega_{x}, \mathbf{y}\right)=\sum_{\mathbf{x}^{\prime} \in \Omega_{x}} e^{\gamma\left(d^{2}(\mathbf{y}, \boldsymbol{\alpha} \cdot \mathbf{x})-d^{2}\left(\mathbf{y}, \boldsymbol{\alpha} \cdot \mathbf{x}^{\prime}\right)\right)}$ and $d^{2}(\mathbf{v}, \mathbf{u})=\sum_{b=1}^{B}|v(b)-u(b)|^{2}$. The expectation $\mathbb{E}_{\mathbf{y} \mid \mathbf{x}}$ can be replaced by an expectation over the noise, $\mathbb{E}_{\mathbf{w}}, w(b) \sim$ $\mathcal{N}(0,1 /(2 \gamma))$. The argument of the exponential functions can be simplified, so that

$$
I\left(\boldsymbol{\alpha}, \gamma, P, \Omega_{z}\right)=\frac{m}{B}-\frac{2^{-m}}{B} \sum_{\mathbf{x} \in \Omega_{x}} \mathbb{E}_{\mathbf{w}}\left[\log _{2}\left(f\left(\boldsymbol{\alpha}, \Omega_{x}, \mathbf{w}\right)\right)\right]
$$

where $f\left(\boldsymbol{\alpha}, \Omega_{x}, \mathbf{w}\right)=\sum_{\mathbf{x}^{\prime} \in \Omega_{x}} e^{-\gamma d^{2}\left(\boldsymbol{\alpha} \cdot \mathbf{x}, \boldsymbol{\alpha} \cdot \mathbf{x}^{\prime}\right)+\sum_{b=1}^{B} f_{2}(b)}$ and $f_{2}(b)=2 \gamma w(b) \alpha_{b}\left(x(b)-x^{\prime}(b)\right)$. This expression can be further simplified by approximating the exponential functions and logarithms by their respective Taylor series for small $\gamma \alpha_{b}^{2}, \forall b=1, \ldots, B$. Next, the expectation of the expression over the random vector $\mathbf{w}$ can be replaced by an expectation over $\gamma \mathbf{w}$, where $\gamma \mathbf{w} \sim \mathcal{N}\left(\mathbf{0}, \frac{\gamma}{2} \mathbf{I}\right)$ (I is the identity matrix) for a fixed $\gamma$. Therefore, we can drop all terms that are proportional to $\mathbb{E}_{\gamma w(b)}[\gamma w(b)], \forall b$, and replace $\mathbb{E}_{\gamma w(b)}\left[(\gamma w(b))^{2}\right]$ in all terms proportional to $\mathbb{E}_{\gamma w(b)}\left[(\gamma w(b))^{2}\right]$ by $\frac{\gamma}{2}$. Now, after some calculus, we obtain

$$
I\left(\boldsymbol{\alpha}, \gamma, P, \Omega_{z}\right)=\frac{\gamma \sum_{b} \alpha_{b}^{2} \operatorname{Var}\left(X_{b}\right)}{B \log (2)}+\sum_{b=1}^{B} o\left(\gamma \alpha_{b}^{2}\right),
$$

where $\operatorname{Var}(X(b))$ is the variance of the $b$-th component of the points of constellation $\Omega_{x}$. The validity of this approximation for small instantaneous SNR has been verified numerically. As the projection of $\Omega_{x}$ on either coordinate axis yields the same set of points, this variance is independent of $b$. Hence, for small instantaneous SNR, the mutual information remains constant for the set of fading points where $\sum_{b=1}^{B} \alpha_{b}^{2}$ is constant. By the definition of $\alpha_{o}$, it is clear that for low SNR, the outage boundary coincides with the hypersurface of the B-hypersphere $\sum_{b=1}^{B} \alpha_{b}^{2}=\alpha_{o}^{2}$.

It is now proved that in both SNR regimes, the outage boundary is outer bounded by the considered $B$-hypersphere. This outer boundary corresponds with an upper bound on the outage probability. Minimizing this upper bound is simply achieved by minimizing $\alpha_{o}^{2}$. Summarizing, the optimal rotation angles are different in both SNR regimes (Fig. fig: high SNR), but are obtained with the same optimization procedure, which is therefore valid for all spectral efficiencies.

\section{Application to CoOperative Communications}

We denote the outage probability optimized above by $P_{\text {out }, p t p}(\gamma)$, where $p t p$ stands for point-to-point. The optimization of the upper bound is covered in [6] and not repeated here. Summarizing, it consist of choosing $P$ so that $\alpha_{o}^{2}=\frac{I_{\mathcal{S}_{p}}^{-1}\left(B R, P, \Omega_{z}\right)}{\gamma}$ is minimized. The mutual information $I_{\mathcal{S}_{p}}($.$) only concerns one subchannel, so that its evaluation$ does not require much computational effort.

We apply Prop. 22 to cooperative communications [15], [9], [10]. Consider for example the relay channel, the most elementary example of a cooperative network. In the case of coded cooperation [9], the relay (R) decodes the message received from the source $(\mathrm{S})$, and then transmits additional parity bits, related to the message, to the destination (D) 3 . The transmission of a codeword is organized in two frames which form together one block. In the first frame of a block, $\mathrm{S}$ broadcasts the first part of the codeword to $\mathrm{R}$ and $\mathrm{D}$. In the second frame, $\mathrm{R}$ cooperates and sends additional parity bits if it is able to decode the transmission of $\mathrm{S}$ in the first frame. Hence, two cases are distinguished, depending on whether $\mathrm{R}$ is able to decode the message from $\mathrm{S}$. We denote the case that $\mathrm{R}$ can decode the message from $\mathrm{S}$ by $c_{1}$ and the other case by $c_{2}$. The S-R channel, S-D channel, and the R-D channel are modelled as memoryless with additive white Gaussian noise and multiplicative real-valued fading $\left(\alpha_{S R}, \alpha_{S D}\right.$ and $\alpha_{R D}$ respectively) which is Rayleigh distributed. The average SNRs

\footnotetext{
${ }^{3}$ We do not claim the optimality of coded cooperation on relay channels, which only illustrates the applicability of our work to cooperative channels.
} 
are denoted by $\gamma_{S R}, \gamma_{S D}$ and $\gamma_{R D}$, respectively. We consider the simple case where $\gamma_{S D}=\gamma_{R D}=\gamma_{u l}$, where $u l$ stands for uplink, and the length of both frames are equal. The fading gains are constant during at least 2 frames. Hence, the outage probability $P_{\text {out }, \text { rel }}$ is

$$
P_{\text {out }, \text { rel }}\left(\gamma_{u l}, \gamma_{S R}\right)=P\left(c_{1}\right) P\left(\text { out } \mid c_{1}\right)+P\left(c_{2}\right) P\left(\text { out } \mid c_{2}\right)
$$

In $c_{1}$, the most favourable case, $\mathrm{D}$ receives both parts of the codeword with constant but independent fading gains, as in a point-to-point BF channel with $B=2$, so that $P\left(\right.$ out $\left.\mid c_{1}\right)=$ $\left.P_{\text {out }, p t p}\right|_{\alpha_{1}=\alpha_{S D}, \alpha_{2}=\alpha_{R D}}\left(\gamma_{u l}\right)$. Hence, the precoding matrix minimizing $P_{\text {out }, p t p}(\gamma)$ also minimizes $P\left(\right.$ out $\left.\mid c_{1}\right)\left(\gamma_{u l}\right)$. Furthermore, $P\left(c_{1}\right)=1-P\left(c_{2}\right)$ and $P\left(c_{2}\right)$ is the probability that $\mathrm{R}$ cannot decode the first part of the codeword, affected by $\alpha_{S R}$, so that $P\left(c_{2}\right)=P\left(I\left(\left.\boldsymbol{\alpha}\right|_{\alpha_{2}=0, \alpha_{1}=\alpha_{S R}}, \gamma, P, \Omega_{z}\right)<R\right)$. Recall that the optimization of $P_{\text {out }, p t p}(\gamma)$ involves the maximization of the mutual information at $\boldsymbol{\alpha}_{o}$, so that this also minimizes $P\left(c_{2}\right)$ and thus maximizes $P\left(c_{1}\right)$. In $c_{2}, \mathrm{D}$ only receives the first part of the codeword, affected by $\alpha_{S D}$, so that the same reasoning as for $P\left(c_{2}\right)$ applies, replacing $\alpha_{S R}$ by $\alpha_{S D}$, so that also $P\left(\right.$ out $\left.\mid c_{2}\right)$ is minimized. The extension to $B-1$ relays for $B>2$ is straightforward.

Summarizing, the precoding matrix minimizing $P_{\text {out }, p t p}(\gamma)$ also minimizes $P_{\text {out }, \text { rel }}\left(\gamma_{u l}, \gamma_{S R}\right)$, if $\gamma_{S D}=\gamma_{R D}=\gamma_{u l}$. This is illustrated in Fig. 4, where $\gamma_{S R}=\gamma_{u l}+5 \mathrm{~dB}$ (other interuser channel conditions yield the same conclusions). The outage probability corresponding to discrete input alphabets is minimized because it approaches the outage probability corresponding to an i.i.d. continuous Gaussian input alphabet very closely.

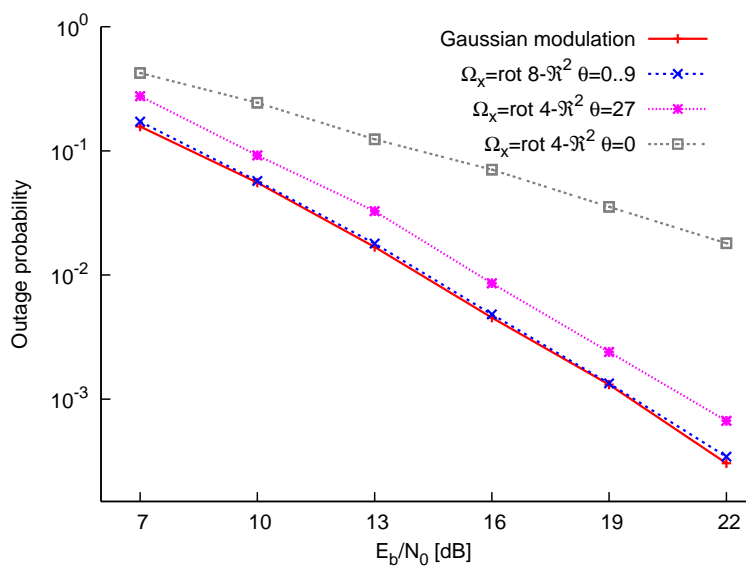

Fig. 4. The outage probabilities of the BF relay channel with real inputs are shown for a fixed spectral efficiency $R=0.9 \mathrm{bpcu}$. The input alphabet is derived from $\Omega_{z}=8-\mathcal{R}^{2}$ or $\Omega_{z}=4-\mathcal{R}^{2}$.

In the case that $\gamma_{S D} \neq \gamma_{R D}$, the optimization procedure has to be slightly modified. Denoting as $b^{\prime}$ the index of the sub- channel with the smallest average SNR, then the hypersurface now only touches the outage boundary at $\boldsymbol{\alpha}_{b^{\prime}, o}$, so that only this magnitude should be optimized.

\section{ACKNOWLEDGEMENT}

The work of Joseph Boutros and part of the work of Dieter Duyck were supported by the Broadband Communications Systems project funded by Qatar Telecom (Qtel). Dieter Duyck and Marc Moeneclaey wish to acknowledge the activity of the Network of Excellence in Wireless COMmunications NEW$\mathrm{COM}++$ of the European Commission (contract n. 216715) that motivated this work.

\section{REFERENCES}

[1] D. Bayer-Fluckiger, F. Oggier, E. Viterbo, "New algebraic constructions of rotated $Z^{n}$-lattice constellations for the Rayleigh fading channel ," IEEE Tr. on Inf. Theory, vol. 50, no. 4, pp. 702-714, 2004.

[2] E. Biglieri, J. Proakis, and S. Shamai, "Fading channels: informationtheoretic and communications aspects," IEEE Tr. on Inf. Theory, vol. 44 no. 6, pp. 2619-2692, Oct. 1998 .

[3] J.J. Boutros and E. Viterbo, "Signal space diversity: a power- and bandwidth-efficient diversity technique for the Rayleigh fading channel," IEEE Tr. on Inf. Theory, vol. 44, no. 4, pp. 1453-1467, July 1998.

[4] J.J. Boutros, A. Guillén i Fàbregas, and E. Calvanese, "Analysis of coding on non-ergodic block fading channels," Allerton Conf. on Communication and Control, Illinois, 2005.

[5] D. Duyck, J.J. Boutros, M. Moeneclaey, "Rotated Modulations for Outage Probability Minimization: a fading space approach," ISIT (Intern. Symp. on Inf. Theory), Texas, June 2010.

[6] D. Duyck, J.J. Boutros, M. Moeneclaey, "Precoded Modulations for Outage Probability Minimization on Block Fading channels," WIC/IEEE SP Symposium on Information Theory and Signal Processing in the Benelux, Brussels, Belgium, May 10-11.

[7] D. Duyck, J.J. Boutros, M. Moeneclaey, "Precoding for Outage Probability Minimization on Block Fading Channels," Submitted to IEEE Tr. on Inf. Theory, March 2011.

[8] A. Guillén i Fàbregas and G. Caire, "Multidimensional coded modulation in block-fading channels," IEEE Tr. on Inf. Theory, vol. 54, no. 5, pp. 2367-2372, 2008.

[9] T.E. Hunter, Coded cooperation: a new framework for user cooperation in wireless systems, Ph.D. thesis, University of Texas at Dallas, 2004.

[10] J.N. Laneman, D. Tse, and G.W. Wornell, "Cooperative diversity in wireless networks: Efficient protocols and outage behavior," IEEE Tr. on Inf. Theory, vol. 50, no. 12, pp. 3062-3080, Dec. 2004.

[11] A. Lozano, A. M. Tulino, and S. Verdú, "Optimum power allocation for parallel Gaussian channels with arbitrary input distributions," IEEE Tr. on Inf. Theory, vol. 52, no. 7, pp. 3033-3051, July 2006.

[12] S.K. Mohammed, E. Viterbo, Y. Hong, and A. Chockalingam, "MIMO Precoding with X- and Y-Codes," IEEE Tr. on Inf. Theory, vol. 57, no. 6, pp. 3542-3566, June 2011

[13] L.H. Ozarow, S. Shamai and A.D. Wyner, "Information theoretic considerations for cellular mobile radio," IEEE Tr. on Veh. Techn., vol. 43, no. 2, pp. 359-379, May 1994.

[14] F. Pérez-Cruz, M.R.D. Rodrigues and S. Verdú, "Optimal precoding for Digital Subscriber Lines," Proc. Intern. Conf. on Comm. (ICC), Beijing, China, May 2008.

[15] A. Sendonaris, E. Erkip, and B. Aazhang, "User cooperation diversity. Part I. System description. Part II. Implementation aspects and performance analysis," IEEE Tr. on Comm., vol. 51, no. 11, pp. 1927-1948, Nov. 2003. 